\title{
GESTIÓN DE RESIDUOS LÍOUIDOS EN LA PRODUCCIÓN DE ACEITE DE OLIVA EN LA EMPRESA DEINAL S.A.C. TACNA PERÚ
}

\section{MANAGEMENT OF LIQUID WASTE IN THE PRODUCTION OF OLIVE OIL IN THE COMPANY DEINAL S.A.C. TACNA PERÚ}

\author{
Carina Alejandra Destéfanis-Petricorena ${ }^{1}$ \\ (D) https://orcid.org/0000-0002-1045-4394 \\ Abel EduARdo Fernandez-MAURIAL ${ }^{2}$ \\ (D) https://orcid.org/0000-0002-9883-8447 \\ MARÍA URSULA CaVERO-ROMAÑa ${ }^{3}$ \\ (D) https://orcid.org/0000-0001-6216-2281 \\ Oscar Alberto Mejia-Sanchez ${ }^{4}$ \\ (D) https://orcid.org/0000-0002-4800-0902
}

Información del artículo: Recibido: 21/01/2021 Aceptado: 01/06/2021 Publicado: 28/06/2021

1,3 Deinal S.A.C Tacna Perú

2,4 Docente en la Escuela de Ingeniería Industrial, Universidad Privada de Tacna E-mail: ${ }^{1}$ cdestefanis@deinalsac.com, ${ }^{2}$ abefernandez@upt.pe, ${ }^{3}$ ursulacavero@gmail.com, 4osamejia@upt.pe 


\section{Resumen}

La producción de aceite de oliva virgen en la Empresa DEINAL S.A.C. se utiliza el proceso de centrifugación de dos fases y por el cual se obtiene el "alperujo", que es el desecho semisólido compuesto por residuos de piel, hueso, pulpa y alto porcentaje de humedad. La generación de grandes cantidades de alperujo que se generan durante la extracción de aceite de oliva, constituye un problema medioambiental, económico y social en el sector olivícola. El objetivo del proyecto es el diseño e implementación de procesos para la gestión de los residuos líquidos en el proceso de extracción de aceite de oliva de la empresa DEINAL S.A.C. El resultado de la implementación de nuevos procesos permitió retirar del efluente líquido los restos de sólidos en suspensión, mediante un separador de grasas adosado a un colector de aceite, que logró aislar de manera eficiente la materia grasa del residuo líquido procedente del proceso productivo, permitiendo la valoración de dicha materia grasa. El residuo líquido libre de residuos sólidos y materia grasa se acumuló en balsas de evaporación.

Palabras Clave: Aceite de oliva, efluente líquido, residuos líquidos, residuos olivícolas.

\section{Abstract}

The virgin olive oil production of the Company DEINAL S.A.C. uses the two-phase centrifugation process and by which the "Alperujo" is obtained, which is the semi-solid waste composed of residues of skin, bone, pulp and high percentage of moisture. The generation of large quantities of Alperujo generated during the extraction of olive oil is one of the greatest environmental, economic and social problems in the olive sector. The objective of the project is the design and implementation of processes for the management of solid and liquid waste in the olive oil extraction process of the company DEINAL S.A.C. The result of the implementation of new processes, allowed the removal of suspended solids from the aqueous effluent by means of a grease separator attached to an oil collector, which was able to efficiently isolate fat from the aqueous residue from the production process, allowing the titration of said fat. The aqueous residue free of solid residues and fat was accumulated in evaporation ponds.

Keywords: Olive oil, liquid effluent, liquid residues, olive residues. 


\section{Introducción}

Hoy en día, al desarrollo de la sociedad se añade el impacto negativo al medio ambiente, el cual es producto de los efectos negativos de la actividad industrial en el mundo. La dimensión de este impacto negativo ha sido preocupante para el ser humano a partir de la última década. A partir de este contexto, el uso inadecuado del recurso hídrico en el sector agroindustrial constituye un alto porcentaje de los costos medioambientales que se traduce en un deterioro del ecosistema, generando la salinización y degradación de suelos. El resultado es una deficiente capacidad en la producción, que conlleva a la escasez de alimentos, entorno difícil en un planeta con un incremento en la población que alcanza los 90 millones de habitantes por año (FAO, 2015). El Perú no es ajeno a esta realidad y lo que urge, es encontrar opciones para una eficiente gestión de los efluentes para su limpieza y recuperación, y de esta forma evitar que su contaminación produzca un daño irreversible.

En el sector agroindustrial, el cultivo del olivo ha experimentado en los últimos 20 años una importante propagación a nivel mundial siendo en la actualidad cultivado en más de 60 países, sin embargo, no solo se ha extendido el cultivo del olivo, sino también la industrialización del olivo, que se caracteriza por una ausencia de técnicas de procesado y aprovechamiento de los residuos, lo que necesita, la implementación de soluciones para reducir el impacto ambiental.

En la industrialización del olivo, el producto primario es el aceite de oliva, y para su elaboración se debe tener en consideración el establecimiento de mecanismos que permitan reducir costos medioambientales.

El sistema de extracción de aceite de oliva en dos fases que desarrolla la empresa DEINAL S.A.C., genera tres residuos diferentes: 1 ) aguas provenientes del lavado del olivo; 2) aguas provenientes de lavado de aceite (centrifugación) y 3 ) residuos sólidos con alto porcentaje de humedad (alperujo). El proceso de extracción de aceite de oliva en dos fases, permite reducir sustancialmente el consumo de agua, lo cual, no exime que se generen efluentes líquidos en el proceso constituidos por el residuo 1 y 2, cuyas cantidades son alrededor del 0,25 L/Kg de olivo procesado (Borja et al. 2006).

El alperujo o residuo generado por la obtención del aceite de oliva aún conserva cierta cantidad de aceite y que puede extraerse mediante un proceso de doble centrifugación y extracción utilizando disolventes, para obtener aceite de orujo y orujillo empleado como combustible. A su vez, el tratamiento de aguas residuales del proceso de extracción de aceite de oliva se extiende a nivel mundial, utilizado ampliamente en países con escasez de recursos hídricos, sumado a que la depuración de efluentes líquidos, es de vital importancia en la protección del medio ambiente responsable, en la totalidad de industrias (Iglesias et al. 2015).

El proyecto, permitió implementar procesos para el tratamiento de los residuos líquidos que se generan en la fabricación del aceite de oliva, en la empresa DEINAL SAC.

\section{Objetivos}

Implementar procesos para el tratamiento de los residuos líquidos que se generan en la fabricación del aceite de oliva, en la empresa DEINAL SAC.

\section{Metodología}

La materia prima utilizada en el presente trabajo ha sido alperujo, proveniente de la Empresa DEINAL S.A.C, Planta de Procesamiento ubicada en el distrito de La Yarada Los Palos en Tacna, Perú. 
Para la implementación de los procesos se han adquirido los siguientes equipos utilizados más significativos:

- Medidores modelo M120 Q3 10.0-R160x40mm (Caudalímetros).

- Bomba EP MIDEX30.

- Electrobomba CST 400/3 Trifásica 220/380 V 60HZ.

- Colectores de aceite de Oil Skimmer

- Colectores de aceite de Oil Grabber

- Coches colectores de aceite de acero inoxidable.

- Difusores.

- Separadores de grasa.

- Filtro de acero inoxidable

Cabe destacar que la Empresa ya contaba con la balsa de acumulación, con una capacidad de $500 \mathrm{~m}^{3}$, tal como se puede observar en la figura 1.

Las acciones llevadas a cabo son:

\section{Medición del consumo de agua en el proceso para el cálculo del vertido}

El registro de consumo de agua en el proceso productivo es de suma importancia, pero, no se conocía el consumo real de agua por proceso, por lo que se procedió a la instalación de caudalímetros, instalándose al inicio de las distintas áreas.

\section{Figura 1}

Balsa de acumulación antes del proyecto.

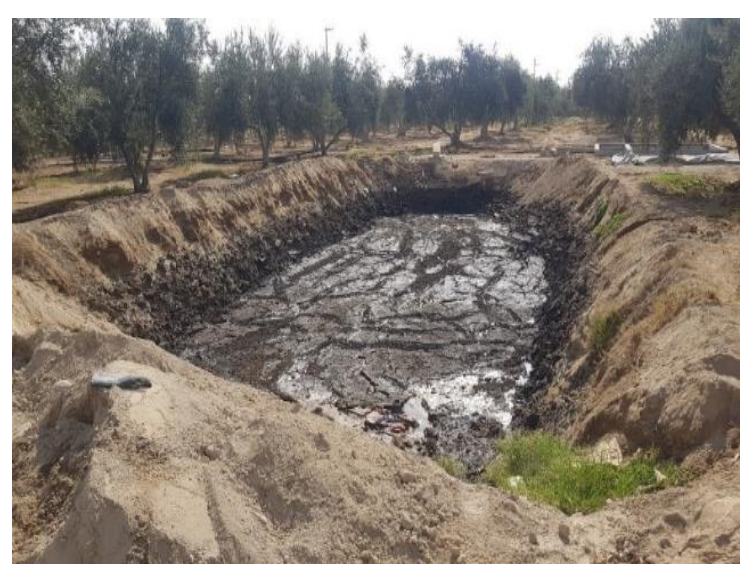

Esta implementación permitió conocer el consumo real de agua por área y disponer de un indicador con el cual comparar el consumo de agua por área de la empresa Descals Industrias Alimentarias SAC (figura 2).

Análisis del proceso de producción. Se realizó un análisis del proceso de producción y del balance de materia en el proceso productivo, lo que permitió disponer de indicadores para evaluar costo/beneficio del rendimiento productivo y producción de efluentes líquidos y sólidos.

Canalización de residuos líquidos. Se procedió a canalizar y entubar con tuberías de PVC, tal como se puede observar en la figura 3, la trayectoria de los residuos líquidos que salen de la planta de procesamiento a la balsa de evaporación, con la finalidad de evitar filtraciones al terreno. Asimismo, se colocaron filtros en los terminales a fin de minimizar el paso de sólidos groseros. 
Figura 2

Bomba presurizada con caudalímetro

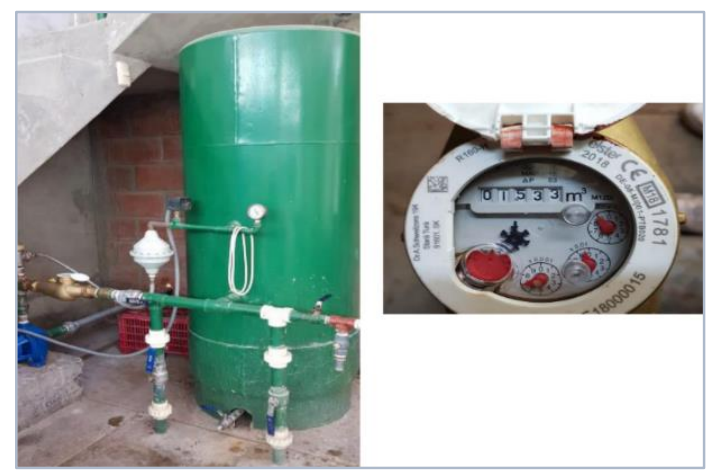

Figura 3

Entubado de los efluentes

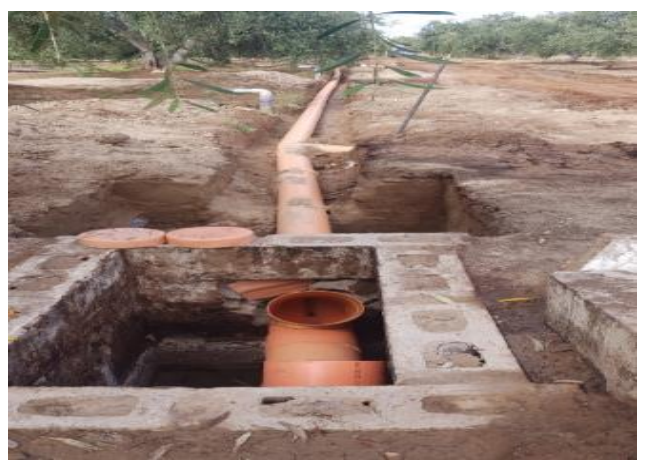

\section{Acondicionamiento e implementación de separadores de grasa}

Se implementó un separador de grasa (como prueba piloto) con la finalidad de evitar el mayor paso de grasas hacia la balsa de acumulación y facilitar la limpieza de sólidos en suspensión. Se construyó el separador de grasa que consiste de 2 piletas de cemento tarrajeadas, intercomunicadas por el fondo, con un tabique inferior de $50 \mathrm{~cm}$ de altura, que permite reducir el paso de sólidos en suspensión hasta la segunda pileta. En esta segunda pileta se instaló un tubo de PVC de $200 \mathrm{~mm}$ de diámetro, con sección exterior en T para inspección en caso de atasco de la cañería. De esta sección, se envía el efluente resultante hacia la balsa de acumulación.

Esta operación se realiza cada vez que en la primera pileta del separador se acumula una capa de grasa de al menos $5 \mathrm{~cm}$ y/o siempre antes de que rebalse. Para realizar esta colección de la grasa superficial se instalaron dos bombas con caño aspirante con colector de aceite Oil Grabber 8 y colector de aceite de manguera Oil Skimmer.

El aceite que se recupera es colectado en coches de acero inoxidable con división central y de allí se bombea el aceite recuperado mediante Bomba EP MIDEX 30 hasta un filtro de acero inoxidable para filtrar los sólidos finos en la recuperación del aceite lampante.

La construcción de los separadores de grasa y la colocación de los colectores en línea ha permitido que el efluente llegue a balsas con una mínima cantidad de grasa y mayormente libre de sólidos en suspensión.

\section{Figura 4}

Separadores de grasa con colectores instalados

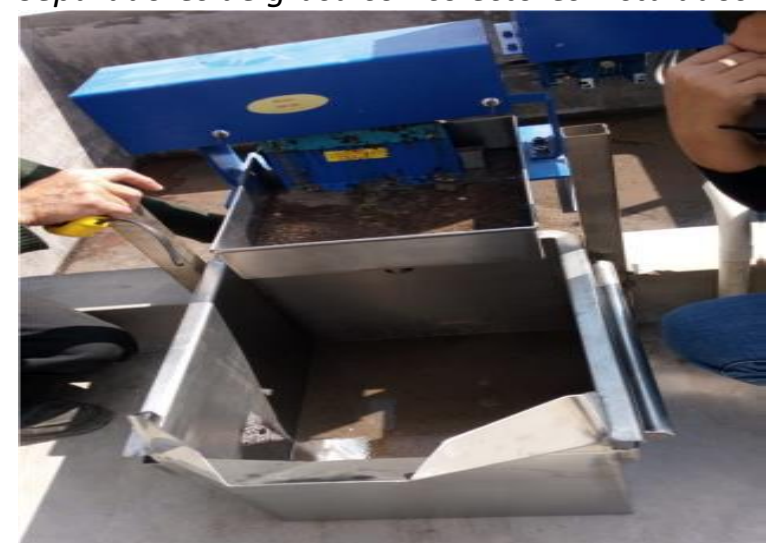

\section{Figura 5}

Balsa de acumulación después del proyecto

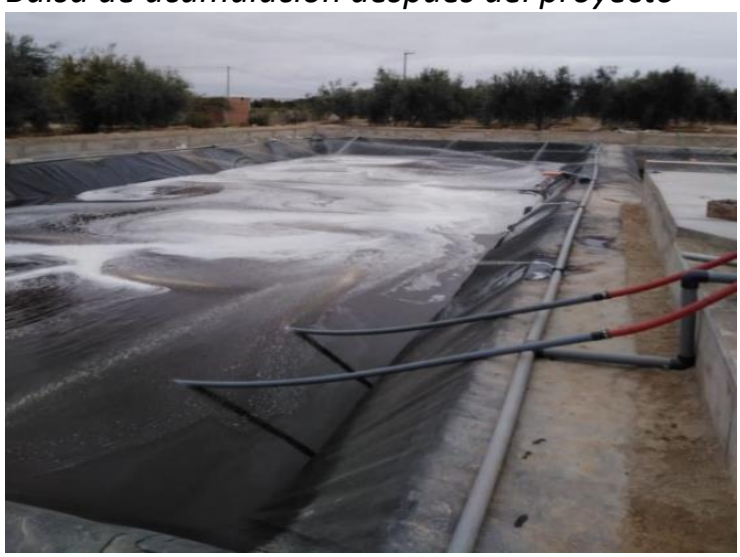


La balsa de acumulación tiene una capacidad de $500 \mathrm{~m}^{3}$, recibe los efluentes líquidos procedentes de los separadores de grasas. Se estima que esta primera balsa acumulará sedimento a lo largo del tiempo, pero no será necesario vaciarla para su limpieza una vez por año. Para ello, se ha adquirido una bomba para impulsar el líquido hasta alcanzar la capa de grasa superficial y una vez que esto ocurra se dejará secar y sacar el sedimento mediante medios manuales o mecánicos.

Figura 6

Esquema general de gestión de efluentes procedentes de industria elaboradora aceite de oliva

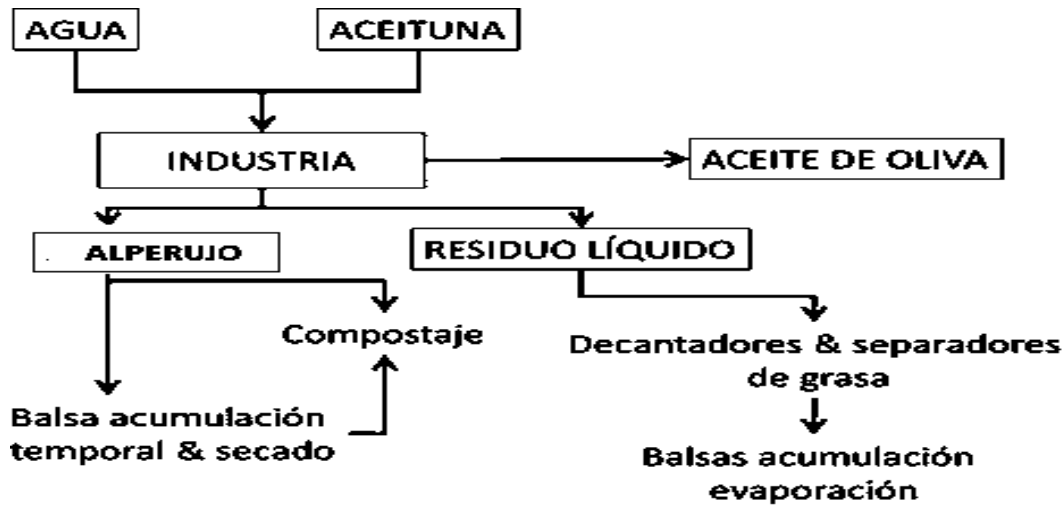

Nota. Flujo desarrollado en Descals Industrias Alimentarias SAC.

\section{Resultados}

La empresa DEINAL SAC al instalar los contadores pudo conocer el consumo real de agua de cada una de las áreas.

La implementación de separadores de grasa con colectores instalados en el proceso de producción, permitieron recuperar aceite lampante en el separador de grasas.

La empresa DEINAL SAC diseñó un proceso para el tratamiento de sus residuos líquidos, resultantes del proceso de producción de aceite de oliva. Proceso que consistió en una primera etapa en la reducción del efluente líquido mediante la optimización del consumo de agua, en una segunda etapa, en la construcción de separadores de grasa a fin de poder separar los sólidos pesados, sólidos ligeros y aceite en suspensión, que permitió pasar un efluente líquido depurado, que con el uso de aspersores que va a facilitar la evaporación del agua en la balsa de evaporación.

\section{Discusión}

Los resultados obtenidos confirman los objetivos propuestos en el estudio, los que coinciden con la mayoría de los estudios similares como ser:

El caudal de agua utilizado antes del desarrollo del proyecto, era mucho mayor y no se conocía la cantidad de agua utilizada en cada una de las áreas de la producción de aceite de oliva, al implementar el proyecto, este caudal de agua fue de 1,67 m3 por tonelada de aceituna y permitió ajustar dicho caudal de 0,5 a 0,8 m3 por tonelada de aceituna.

Los separadores de grasa instalados en el proceso de producción permitieron que de un valor promedio de 8000 L/día de efluentes líquidos producidos en la empresa, se logró recuperar un 0,3\% de aceite lampante en el separador de grasas. 
El aceite de oliva que era enviado a la balsa se recuperó en su totalidad, lo que redundará en un beneficio económico.

\section{Conclusiones}

La gestión de efluentes líquidos procedentes de la elaboración de aceite de oliva en la empresa DEINAL SAC, mejoraron mediante la modificación de su proceso productivo en las distintas áreas de producción.

Los beneficios que se alcanzaron tras la implantación de estas modificaciones son:

- Recuperación del aceite de oliva que era enviado a la balsa.

- Reducción del total de efluente líquido contaminante acumulado en balsas y reducción de los olores producidos, lo que reportará un beneficio medioambiental

- Por último, la capacidad de reducir el volumen de efluente contaminante, y por encima de eso, la posibilidad de incorporar a la cadena productiva los actuales residuos, hacen de este nuevo sistema de gestión un ejemplo a seguir dentro de los actuales sistemas de economía verde o economía circular.

\section{Referencias bibliográficas}

Borja, R., Raposo, F., \& Rincón, B. (2006). Treatment technologies of liquid and solid wastes from twophase olive oil mills. Grasas y aceites, 57(1), 32-46.

Iglesias, F. A. C., García, C. M., Palomino, T. C., Godino, F. J. I., \& De Linares, E. P. S. Residuos y subproductos. Oportunidad de negocio. Ejemplo en la extracción del aceite de oliva en Andalucía (España).

Descals Industrias Alimentarias SAC. (2019). Diseño de planta piloto para la gestión más eficiente de los residuos líquidos procedentes de industria elaboradora de aceite de oliva. [Archivo].

Descals Industrias Alimentarias SAC. (2019). Análisis de los procesos de producción de la empresa e identificación de cuellos de botella en cuanto al tratamiento de residuos sólidos y líquidos. [Archivo].

Destefanis, C., y Fernández, A. (2019). Implementación de la solución tecnológica para el tratamiento de los residuos sólidos y líquidos generados en los procesos industriales de la empresa Descals Industrias Alimentarias SAC (Deinal SAC). [Archivo].

Descals Industrias Alimentarias SAC. (2019). Informe sobre Prospectiva Industrial de tecnologías disponibles para el tratamiento de los efluentes sólidos y líquidos. [Archivo]. 